\title{
30 AN ELECTRONIC MARKET ARCHITECTURE FOR THE FORMATION OF VIRTUAL ENTERPRISES
}

\author{
Ana P. Rocha", Eugénio Oliveira \\ University of Porto, Portugal
}

\begin{abstract}
Economic organisations are facing new challenges due to the proliferation of Electronic Markets where competition and responsiveness are issues that will determine organisation's economic survival. A Virtual Enterprise is a temporary network of individual enterprises, which, due to its higher flexibility and agility is capable of effectively responding to those challenging requirements. This paper proposes an Electronic Market system's architecture together with a multi-criteria negotiation protocol for Virtual Enterprise formation.
\end{abstract}

\section{INTRODUCTION}

A Virtual Enterprise (VE) is an aggregation of autonomous and independent enterprises connected through a network (possibly a public network like WWW) and brought together to deliver a product or service in response to a customer need. Virtual Enterprise management should be supported by efficient information and communication technology. This aggregation of enterprises is advantageous in the sense that it will reduce complexity - today's products and services are increasingly complex and requires close coordination across many different disciplines - and most important, will enable the response to rapidly changing requirements. The Virtual Enterprise will only exist for a temporary time limit, that is the time needed to satisfy its purpose.

The VE life cycle is decomposed in four phases (Faisst, 1997) (Fischer, 1996), namely:

- Identification of Needs: Description of the product or service to be delivered by the VE, which guides the conceptual design of the VE.

- Partners Selection: Rational selection of the individual enterprises (partners) which will compose the VE, based in its specific knowledge, skills, resources, costs and availability.

\footnotetext{
- University of Porto, LIACC - Faculty of Engineering, Rua dos Bragas, 4000 Porto, Portugal, e-mail: \{arocha,eco\}@fe.up.pt
} 
- Operation: Control and monitoring of the partners' processes, including resolution of conflicts, and possible VE reconfiguration due to partial failures.

- Dissolution: Breaking up the VE, distribution of the obtained profits and storage of relevant information.

Our work is currently focused on the second above mentioned phase (partners selection), and our proposal concerns the development of a Multi-Agent System (MAS) architecture to model the Electronic Market that supports the automatization of the VE.

An Electronic Market (Bakos, 1998) can be seen as the virtual marketplace where business participants that are geographically distributed and possibly not known in advance can meet each other and cooperate in order to achieve a common business goal. An electronic network is used as an information and communication infrastructure, in order to link these distributed entities. In our particular case, business participants are all the individual enterprises linked to the network and the common business goal is the formation of a VE capable of satisfying a specific consumer's need.

In this paper, we propose the use of intelligent agents and multi-agent technology as a framework for the establishment of a VE. In the following section, we will introduce our proposed MAS architecture to model the Electronic Market, focusing our description in the agent's structure and behaviour. A brief survey on agent-based architectures for electronic commerce can be found in (Guttman, 1998). Then we describe a negotiation protocol that includes both multi-criteria evaluation functions and constraint-based procedures that permit a global agreement about the structure of a VE. We finalise the paper with some conclusions and directions for future work.

\section{ELECTRONIC MARKET ARCHITECTURE}

Economic organisations are forced to respond to increasingly more complex and rapidly changing requirements. Virtual Enterprises can satisfy these new challenges, as they combine the core competencies of several autonomous and heterogeneous enterprises aggregated in a temporary network, presenting high flexibility and agility.

An Electronic Market infrastructure can efficiently support VE formation and operation since it constitutes a virtual marketplace where business participants can meet each other and cooperate in order to achieve a common business goal. In our proposed Electronic Market, autonomous enterprises are the business participants and the common business goal is the formation of a VE as the most favourable group of enterprises that can satisfy a specific need. Besides individual enterprises, other participants operate in the Electronic Market, whose role is to facilitate a set of auxiliary market functionalities like searching goods or participants, filtering information or helping negotiation.

Agents (Faratin, 1998) are autonomous or semi-autonomous entities that can perform tasks in complex and dynamically changing environments. Agent technology seems to be an appropriate paradigm for use in modelling individual 
enterprises that participate in the Electronic Market, since they exhibit some relevant capabilities like autonomy, adaptability and ability to interact with others.

A Multi Agent System (MAS) - see also (O'Hare 1996) for a general surveyconsists of a group of agents that combine their specific competencies and cooperate in order to achieve a common goal. Efficient cooperation as well as coordination procedures between agents endow a MAS with a capability higher than the sum of the individual agent's capabilities.

We therefore propose a Multi-Agent System architecture to model the Electronic Market that supports the establishment of the VE (see Figure 1). This MAS includes several heterogeneous and semi-autonomous intelligent agents representing the several independent enterprises, and also a special agent called Market Agent that helps in the VE formation process. We will now describe in more detail the structure and functionality of these agents.

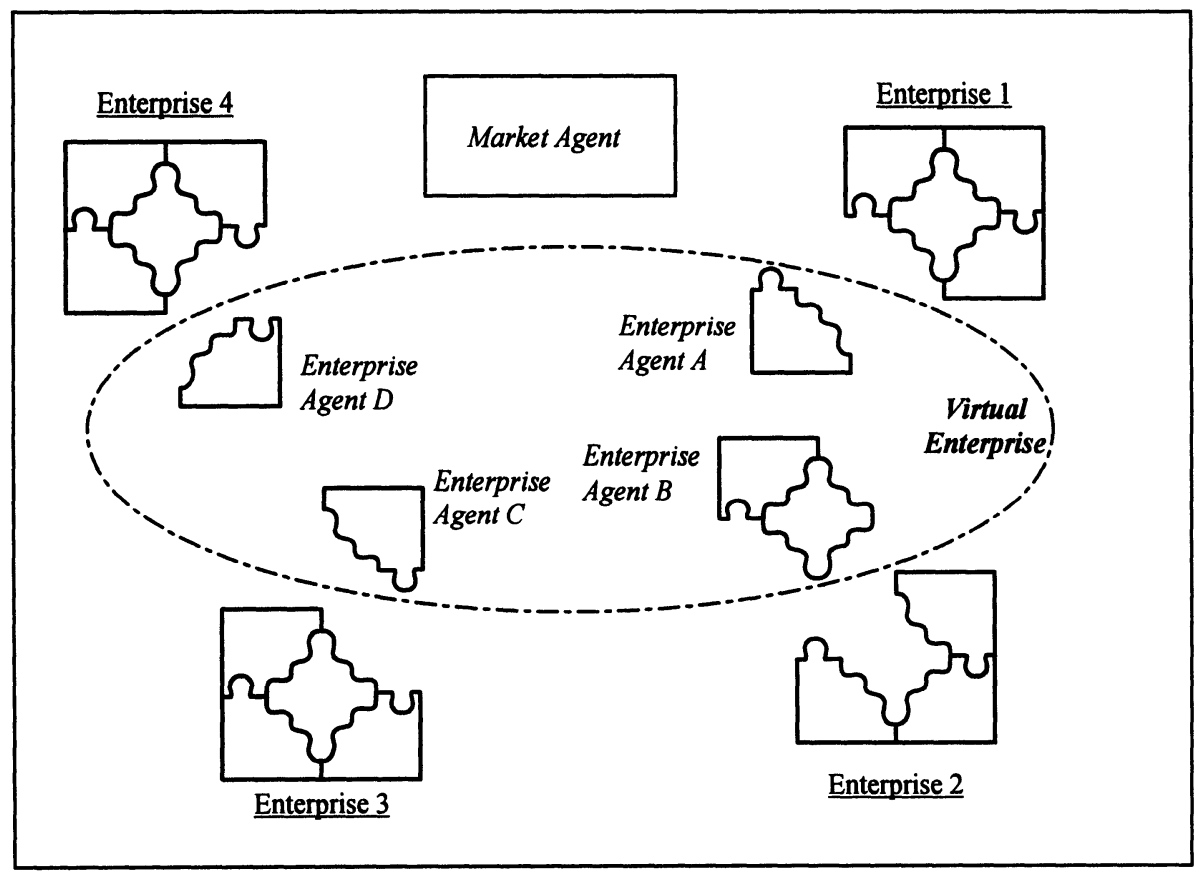

Figure 1 - Electronic Market architecture

\section{Market Agent}

When a specific consumer's need is identified (a consumer being either a human or an enterprise), a new special agent, which is called Market Agent, is created and included in the Electronic Market system. This agent plays the role of coordinator in the Electronic Market where it is placed and its main goal is the Virtual Enterprise formation. The Market Agent structure includes two principal modules: "Goal Descriptor" and "Virtual Enterprise Selector" (see Figure 2). 


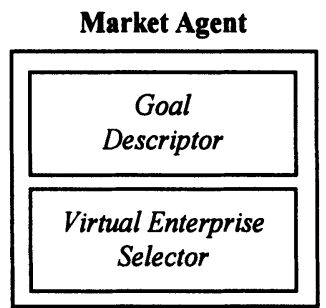

Figure 2 - Market Agent structure

We assume, for now, that the "Goal Descriptor" module is instantiated by the consumer. This module includes the description of the goal to achieve, which is decomposed in a set of sub-goals. Each sub-goal (SGD) is described by means of a deadline and price value, and also a list of attributes with associated utility values. These attributes' values may not be absolute values but instead they may be restricted to a set of preferable values in pre-defined domains. The sub-goals themselves may be related to each other through constraints attached to their own attribute values. A sub-goal description is then represented by the following structure:

\section{$S G D=($ SGId $, L A t, D, P)$}

SGId: Sub-goal identification.

D: Deadline for sub-goal achievement.

P: $\quad$ Price that the consumer is willing to pay for the satisfaction of that specific sub-goal.

LAt: List of evaluation attributes. Each element of the list has the following structure:

$\mathrm{At}_{\mathrm{i}}=($ AtId, PrefV, Dom, Ut $)$

AtId: Attribute identification.

PrefV: Preferable attribute value.

Dom: Attribute values domain.

Ut: Attribute utility (representing the relative importance of the attribute).

The "Virtual Enterprise Selector" module is responsible for the rational selection of the VE partners. In a first step, a set of invitations (one for every subgoal described in "Goal Descriptor" module) is send to the Electronic Market system. In a second step, the received proposals are evaluated and the senders of the most favourable ones are selected and committed as partners of the VE. The VE partners selection is done through multi-criteria evaluation and constraint based negotiation protocols described later in this paper.

\section{Enterprise Agent}

In order to be competitive in today's economic markets, enterprises need not only to be efficient in their business field, but also to be able to rapidly react and adapt to new environments as well as to interact with other enterprises. The control 
architecture adopted for the design of an enterprise agent should meet these requirements. Figure 3 presents our proposed enterprise agent structure.

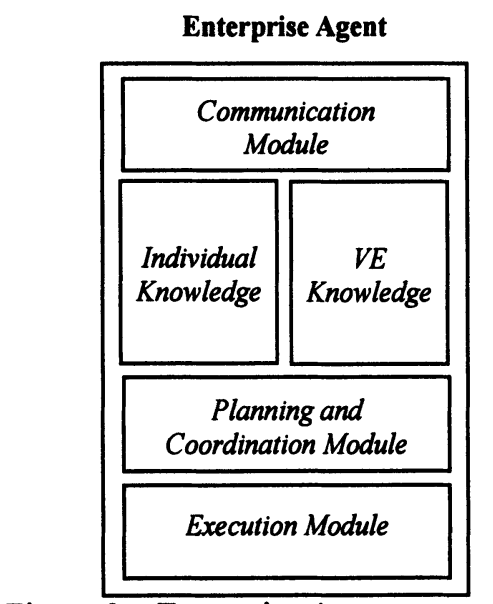

Figure 3-Enterprise Agent structure

An Enterprise Agent's structure comprises three functional modules: Communication, Planning and Coordination, and Execution modules, and two knowledge-based modules: Individual Knowledge and Virtual Enterprise Knowledge module.

The "Communication module" is responsible for all processes related with messages handling. Incoming messages are filtered and the ones that are not relevant for this specific agent are ignored. The relevant messages are then ordered by degree of importance. Outcome messages are sent out only to those agents that are known to be possibly interested in that particular piece of information.

The "Planning and Coordination module" is the most complex one. Its role is to manage local tasks and cooperative behaviour. In our current presentation, the most important issue of this module includes the process to formulate a bid in response to an announcement sub-goal (e.g., a proposal for becoming a VE partner).

The "Execution module" is the intelligent system responsible for the realisation of local tasks an agent is committed to.

Information related to organisational and operational rules defined by the VE, is included in "Virtual Enterprise Knowledge". This module contains also information concerning individual enterprise's rights and duties.

The "Individual Knowledge" contains information about the agent itself: agent's own capabilities and current workload, as well as information related to other agents: other agents commitments and capabilities.

The Enterprise Agent is a template supplied by the Electronic Market that, once being filled in according to the individual enterprise's specific tactics and strategies, will represent that particular enterprise in the market.

Although only the VE formation issue is being discussed in this paper, the Enterprise Agent structure here proposed is able to support all phases of the VE life cycle. 


\section{NEGOTIATION PROTOCOL}

Several examples of intelligent agent-based systems suitable for electronic market operations already exist (Chavez, 1996) (Tsvetovatyy, 1997). Moreover, electronic market functionalities available in existing systems, vary from searching for goods to either a simple purchasing or selling goods, or even to more complex negotiation of goods. It is this negotiation process between participants in an electronic market that becomes our main concern in this paper.

Auctions (Vulkan, 1998), game theory (Rosenschein, 1994) and intelligent agent technology (Jennings, 1996) are paradigms used in many automated negotiation systems. Auction mechanisms are very popular due to its simplicity and well predefined rules, but involves only single criteria negotiations. Game theory mechanisms can only be applied in contexts that present perfect information and perfect rationality. Intelligent agent technology is a more flexible paradigm suitable for dynamic and open environments, since agents can effectively cope with the complexity and large amount of information. Intelligent agent technology seems to be an appropriate paradigm to use in our case, since VE operates in dynamic and complex environments.

A VE combines competencies derived from several heterogeneous and independent enterprises present in the Electronic Market. A negotiation protocol has to be defined in order to select the individual enterprises that will become members of the VE based on its capabilities and availability. In VE formation process, two important issues has to be considered:

- A multi-criteria evaluation is used to select the most favourable candidates: lower cost, higher quality, higher availability, etc

- Enterprise's competencies may inter be related by constraints attached to their attributes values which creates a kind of distributed constraints problem.

Attaching utility values to different criteria solves the problem of multi-criteria evaluation. An evaluation formula is a linear combination of the current attributes' values weighted by their corresponding utility values (Oliveira, 1999).

Distributed Constraint Satisfaction Problems (Yokoo, 1992) are defined by a set of variables $\left(x_{1}, x_{2}, \ldots, x_{n}\right)$ which can take values from domains $D_{1}, D_{2}, \ldots, D_{n}$ respectively, and a set of constraints on their values. Here, in our scenario, variables are distributed among enterprises in the electronic market and instantiations of the variables must satisfy these inter-enterprise constraints.

In this paper we propose a multi-criteria negotiation protocol that includes resolution of constraint-based problems for the VE formation.

The Market Agent plays the role of coordinator in the VE formation process through its "Virtual Enterprise Selector" module. This agent knows the VE subgoals that are described in the "Goal Descriptor" module. For each of them sends out a request message to all enterprises present in the Electronic Market. Received bids are then evaluated using a multi-criteria function and the most favourable are selected. Those enterprises that have sent winning proposals become VE members and are committed to the respective goal achievement. Meanwhile, if different bids include attributes' values that are incompatible, the respective enterprises' agents 
activate a negotiation process searching for an agreement through a set of compatible values.

\section{Request formulation}

The Market Agent formulates a request for each of the VE sub-goals that are described in its "Goal Descriptor" module. These request messages are delivered to all enterprises existing in the Electronic Market, and are represented by the following structure:

\section{request (MAg, Gid, LAt, D, P)}

MAg: Market Agent identification.

Gid: Goal identification.

D, P: Deadline and price value for goal achievement.

LAt: List of evaluation attributes. Each element of the list presents the following structure:

$A t_{i}=($ AtId, PrefV, Dom $)$

AtId: Attribute identification.

PrefV: Preferable attribute value.

Dom: Attribute values domain.

\section{Bid formulation}

In response to a request message, the Enterprise Agent formulates a bid based in its own capabilities and current availability (described in "Individual Knowledge" module). This proposal message is sent out to the Market Agent, and is represented by the following structure:

\section{proposal (EAg, Pid, Gid, LAt, T, P)}

EAg: Enterprise Agent identification.

Pid: Proposal identification.

Gid: Goal identification.

T, P: Time and cost for goal achievement.

LAt: List of attributes' values. Each element of the list presents the following structure:

$$
A t_{i}=(\text { AtId, } V)
$$

AtId: Attribute identification.

V: $\quad$ Attribute value.

Some constraints on other sub-goals' attribute values may result from accepting a particular agent's bid. As an example, suppose that a VE has to be formed in order to deliver a specific type of car. The attribute "dimension" of the component "wheel" (to be able to deliver a wheel is described as a specific goal of a supplier enterprise) may restrict the values of the attribute "material" of the component "screw". This information is sent out to the Market Agent in one or possibly more messages represented by the following structure: 
constraint ( EAg, Pid, At, CAt)

EAg: Enterprise Agent identification.

Pid: Proposal identification.

At : Attribute that constrains other attributes

( AtId, V)

AtId: Attribute identification.

V: $\quad$ Attribute value.

CAt: Constrained attribute

( CGId, CAtId, CDom )

CGId: Identification of the constrained goal.

CAtId: Identification of the constrained attribute.

CDom: Possible values for the constrained attribute.

\section{Bids evaluation}

The "Virtual Enterprise Selector" module evaluates all admissible sets of bids, based in a multi-criteria function. A bids set is admissible if:

- It constitutes a solution, i.e., the bids set can satisfy all the VE sub-goals.

- The attributes' values are mutually compatible.

The multi-criteria function used to evaluate the bids set is the following:

$$
\begin{aligned}
& \sum_{k=1}^{n} \sum_{i=1}^{a t_{k}} \operatorname{dif}\left(\operatorname{Pref} V_{i}, V_{i}\right)^{*} U t_{i} \\
& \text { where } \quad \begin{array}{l}
\mathrm{n}=\text { number of VE sub-goals. } \\
\mathrm{at}
\end{array} \mathbf{t}_{\mathrm{k}}=\text { number of attributes for sub-goal } \mathrm{k} .
\end{aligned}
$$

In the formula above, the function $\operatorname{dif}\left(\operatorname{Pref} V_{i}, V_{i}\right)$ quantifies for an attribute $i$, the degree of acceptability of the current value $\left(V_{i}\right)$ when compared to its preferable value (PrefVi). If the attribute values domain contains numeric values, this quantification is simply a normalised difference between the two values $V_{i}$ and PrefV $V_{i}$. If the attribute values domain contains non-numeric values, the result of the $\operatorname{dif}\left(\operatorname{Pref} V_{i}, V_{i}\right)$ function is now calculated as a normalised difference between the two scoring values attached to $V_{i}$ and $\operatorname{Pref} V_{i}$. The scoring values have been defined together with the attribute values domain and are included in "Goal Descriptor" module.

The winner bids set is selected as the one that presents, by order of preference:

- Lowest evaluation value. The solution includes attributes values closer to the preferable values.

- Lowest cost.

- Lowest conclusion time.

- Lowest number of distinct VE partners. VE operation complexity increases with the number of distinct enterprise partners.

Enterprises that have sent the winning bids are informed that they are included in and committed to the new created VE.

If an admissible bids set cannot be found because some attributes values are mutually incompatible, a constraint satisfaction resolution process is activated. This process is described in the following section. 


\section{Constraint satisfaction problem resolution}

If the "Virtual Enterprise Selector" module receives bids that include mutually incompatible attributes' values, it sends out that information to the respective constrained agents (see Figure 4) using the following message structure:

constraint_to ( Pid, CAt, EAg, At, C)

Pid: Identification of the proposal sent by this agent that presents incompatible attributes' values.

CAt: Constrained attribute.

( CGId, CAtId, CDom )

CGId: Identification of the constrained goal.

CAtId: Identification of the constrained attribute.

CDom: Possible values for the constrained attribute.

EAg: Agent that presents attributes values incompatible with this agent.

At: Constraining attribute imposed by agent $E A g$.

( GId, AtId, V)

GId: Identification of the constraining goal.

AtId: Identification of the constraining attribute.

V: Constraining attribute value.

C: Additional cost for agent $E A g$ to propose the attribute value $V$. This parameter represents the difference between the cost of ensuring either the current value $V$ or the preferable value. We use the difference value and not the cost value itself in order to maintain agent privacy.

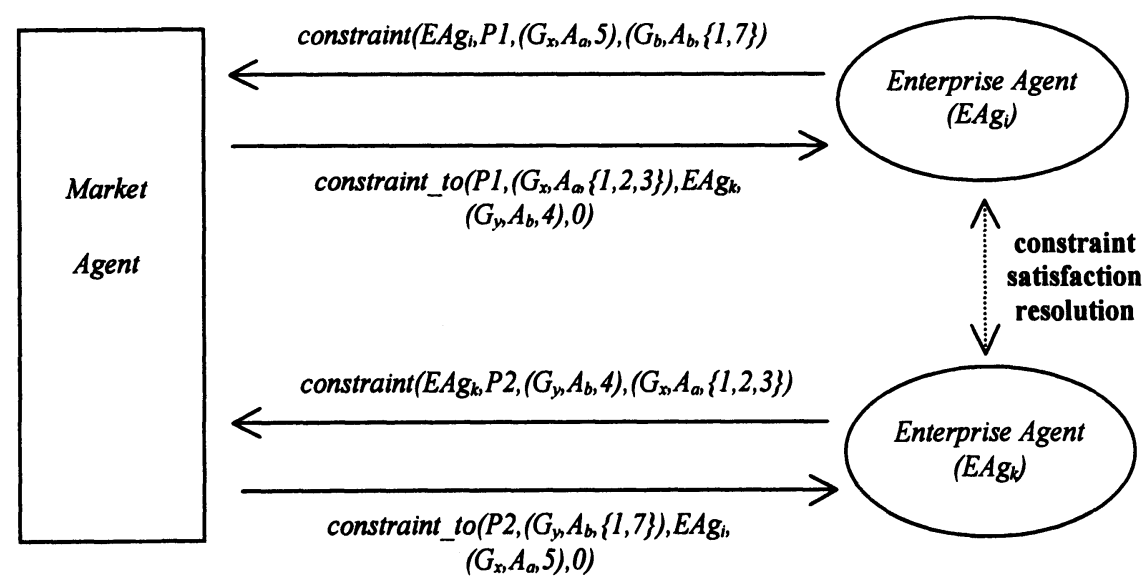

Figure 4 - Constraint satisfaction problem resolution

The Market Agent job is to put in contact constrained agents. It does not participate any more in the constraint problem resolution. Constrained agents negotiate between them in order to agree in a set of admissible values for their constrained attributes.

An agent matches the constrained attributes with all admissible values and selects the solution that is most favourable both to him and, at the same time, to the 
other agents included in this process since the final solution is necessarily a composition of individual solutions. When those agents reach an agreement, the final solution is sending out to "Market Agent".

Next we describe the procedure adopted in the resolution of the distributed constraint satisfaction problem. Let us suppose that there exist two agents in the Market: $\mathrm{Ag}_{\mathrm{i}}$ and $\mathrm{Ag}_{\mathrm{k}}$. $\mathrm{Ag}_{\mathrm{k}}$ sends a constraint message to $\mathrm{Ag}_{\mathrm{i}}$, as described:

constraint_to (Pid, (CGId, CAtId, CDom), Ag $g_{k}$ (GId, AtId, V), C)

If this round is not the first one in the current negotiation process, one more message is receive by $\mathrm{Ag}_{\mathrm{i}}$. This message contains the cost of the last proposal sent to $\mathrm{Ag}_{\mathrm{k}}$ :

last_proposal (Vals, TC_last)

- Solution 0 is the best solution already found, and its cost is TCO

- If TC_last $<\mathrm{TC} 0$ then

$$
\frac{\text { solution } 0}{\mathrm{TC} 0=\mathrm{TC} \text { _last }}
$$

- For all possible values defined in the received message (CDom), it selects the most favorable (solution 1):

$$
\begin{aligned}
& \text { selected value }=\mathrm{V} 1 \\
& \text { cost of achieving } \mathrm{V} 1=\mathrm{Cl} \\
& \text { total solution cost }=\mathrm{Cl}+\mathrm{C}=\mathrm{TC} 1
\end{aligned}
$$

If all values (CDom) are impossible to obtain then $\mathrm{TCl}=$ null

- $\mathrm{Ag}_{\mathrm{i}}$ sends a message to $\mathrm{Ag}_{\mathrm{k}}$ :

last_proposal (\{(GId, AtId, V),(CGId, AtId, V1)\}, TC1)

- For all possible values not yet proposed to agent $\mathrm{Ag}_{\mathrm{k}}$, it selects the most favourable (solution 2):

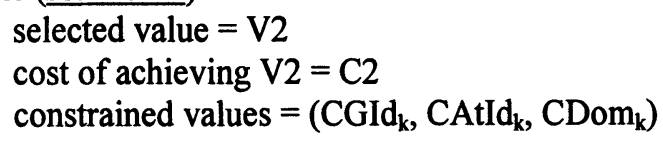

- The solution that has the lowest cost (among TC0, TC1, C2) is selected as the one to send to $\mathrm{Ag}_{\mathrm{k}}$. If the selected solution is either solution 0 or solution $\underline{1}, \mathrm{Ag}_{\mathrm{k}}$ knows that this solution has already been proposed and terminates the negotiation process. If the selected solution is solution 2, the negotiation process goes on. 


\section{CONCLUSIONS AND FUTURE WORK}

Virtual Enterprises are of increasing importance mainly due to its flexibility, agility and efficiency. These main characteristics enable VE successful behaviour in dynamic and complex business environments.

This paper was focused in the use of intelligent agent technology for VE formation. The VE formation process was done through a negotiation protocol that includes resolution of multi-criteria and distributed constraint-based problems. A future improvement we intend to make is to include in our enterprise agents the capability of learning. Learning what is the best strategy to use in a specific negotiation process would be of most benefit for the agents representing enterprises in the Electronic Market.

A Multi-Agent System was also proposed as a support for the implementation of the Electronic Market where individual enterprises meet each other, negotiate and cooperate.

Besides VE formation, distributed agent technology can also help in VE operation. VE operation will further request the monitoring of distributed activities and will possibly include the need of conflict resolution procedures.

\section{REFERENCES}

1. Bakos Yannis. The Emerging Role of Electronic Marketplaces on the Internet. Communications of the ACM, Vol. 41, No. 8, pp. 35-42, August 1998.

2. Chavez Anthony, Maes Pattie. Kasbah: An Agent Marketplace for Buying and Selling Goods. Proceedings of the First International Conference on the Practical Application of Intelligent Agents and Multi Agent Technology, London, UK, April 1996.

3. Faisst Wolfgang. Information Technology as an Enabler of Virtual Enterprises: A Life-Cycle-Oriented Description. Proceedings of the European Conference on Virtual Enterprises and Networked Solutions. Paderborn, Germany, April 1997.

4. Faratin Peyman, Sierra Carles, Jennings Nick R. Negotiation Decision Functions for Autonomous Agents. International Journal of Robotics and Autonomous Agents, Vol. 24, Nos. 3-4, pp. 159-182, 1998.

5. Fischer Klaus, Muller Jorg, Heimig Ingo, Scheer A-W. Intelligent Agents in Virtual Enterprises. Proceedings of the First International Conference on the Practical Application of Intelligent Agents and Multi Agent Technology. London, UK, April 1996.

6. Guttman Robert H., Moukas Alexandros G., Maes Pattie. Agent-Mediated Electronic Commerce: A Survey. Knowledge Engineering Review, June 1998.

7. Jennings N. R., Farantin P., Johnson M. J., O'Brien P., Wiegand M.E. Using Intelligent Agents to manage Business Processes. Proceedings of the First International Conference on the Practical Application of Intelligent Agents and Multi Agent Technology. London, UK, April 1996.

8. O'Hare G. M. P., Jennings N. R., Foundations of Distributed Artificial Intelligence, John Wiley \& Sons, 1996

9. Oliveira Eugénio, Fonseca José M, Steiger-Garção A. Multi-criteria negotiation on Multi-Agent Systems. Proceedings of the First International Workshop of Central and Eastern Europe on MultiAgent Systems, St. Petersburg, Russia, June 1999.

10.Rosenschein Jeffrey S., Zlotkin Gilad. Rules of Encounter. MIT Press, 1994. 
11.Tsvetovatyy Maksim, Gini Maria, Mobasher Bamshad, Wieckowski Zbigniew. MAGMA: An AgentBase Virtual Market for Electronic Commerce. Journal of Applied Artificial Intelligence, Special issue on Intelligent Agents, Vol. 11, No. 6, 1997.

12. Vulkan Nir, Jennings Nick R. Efficient Mechanisms for the Supply of Services in Multi-Agent Environments. Proceedings of the First International Conference on Information and Computation Economics. Charleston, South Carolina, October 1998.

13. Yokoo Makoto, Durfee Edmund, Ishida Toru, Kuwabara Kazuhiro. Distributed Constraint Satisfaction for Formalizing Distributed Problem Solving. Proceedings of the Twelfth IEEE International Conference on Distributed Computing systems, June 1992. 